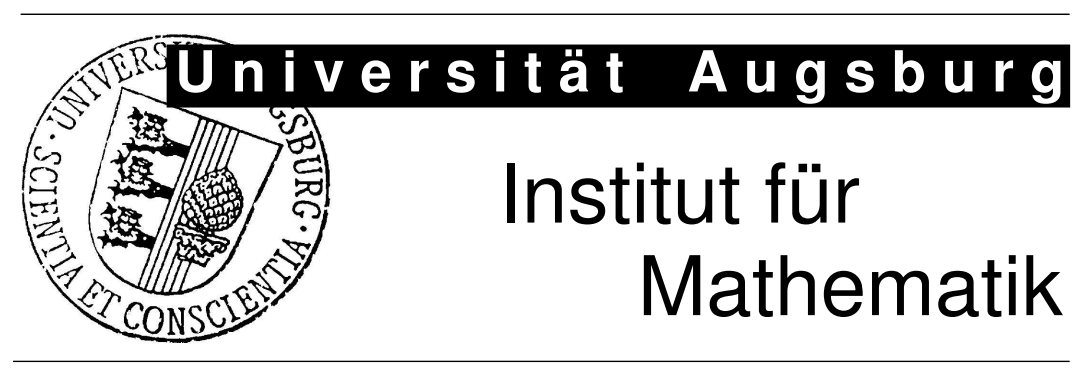

Lothar Heinrich, Stella Klein

Central Limit Theorems for Empirical Product Densities of Stationary Point Processes 


\section{Impressum:}

\section{Herausgeber:}

Institut für Mathematik

Universität Augsburg

86135 Augsburg

http://www.math.uni-augsburg.de/pages/de/forschung/preprints.shtml

\section{ViSdP:}

Stella Klein

Institut für Mathematik

Universität Augsburg

86135 Augsburg

Preprint: Sämtliche Rechte verbleiben den Autoren (C) 2011 


\title{
Central limit theorems for empirical product densities of stationary point processes
}

\author{
Lothar Heinrich and Stella Klein*
}

\begin{abstract}
We prove the asymptotic normality of kernel estimators of second- and higher-order product densities (and of the pair correlation function) for spatially homogeneous (and isotropic) point processes observed on a sampling window $W_{n}$ which is assumed to expand unboundedly in all directions as $n \rightarrow \infty$. We first study the asymptotic behavior of the covariances of the empirical product densities under minimal moment and weak dependence assumptions. The proof of the main results is based on the Brillinger-mixing property of the underlying point process and certain smoothness conditions on the higher-order reduced cumulant measures. Finally, the obtained limit theorems allow to construct $\chi^{2}$-goodness-of-fit tests for hypothetical product densities.

Keywords Kernel-type product densities estimators - empirical pair correlation function · Brillinger-mixing point processes · reduced cumulant measures · large domain statistics $\cdot \chi^{2}$-goodness-of-fit tests
\end{abstract}

Mathematics Subject Classification (2000) $\quad$ 60G55 $62 \mathrm{M} 30 \cdot 60 \mathrm{~F} 05 \cdot 62 \mathrm{G} 20$

\section{Introduction}

The second-order statistical analysis of stationary and isotropic spatial point processes is mainly based on empirical versions of second-order characteristics such as Ripley's $K$-function and the pair correlation function, see e.g. Baddeley et al. [1], Cressie [3], Diggle [6], Illian et al. [10], and Stoyan et al. [16]. Compared with the pair correlation function, the second-order product density contains additional information if the stationary point process is anisotropic. The asymptotic behavior of these estimators has already been studied in, e.g., Jolivet [12], Heinrich [7], and Heinrich and Liebscher [8]. For Poisson cluster processes Heinrich [7] proves a central limit theorem (CLT) for the empirical second-order product density. Heinrich and Liebscher [8] prove almost sure convergence (with rates) of kernel-estimators of the secondorder product density and the pair correlation function for $\beta$-mixing point processes. Jolivet [12] studies the speed of $\mathcal{L}_{p}$-convergence of empirical product densities of any order, and

${ }^{*}$ Lothar Heinrich, Stella Klein : Institut für Mathematik, Universität Augsburg, 86135 Augsburg, Germany email: lothar.heinrich@math.uni-augsburg.de 
sketches the proof of their asymptotic normality at any fixed argument for Brillinger-mixing point processes by deriving sufficiently sharp bounds of the corresponding cumulants of order $k \geq 3$. However, the assumptions stated in Jolivet [12] are not sufficient to get these bounds which is first noticed when determining the exact asymptotic order of the variances. In the present paper we will provide conditions in addition to Brillinger-mixing which are sufficient to hold asymptotic normality of the joint distribution of empirical product densities (and likewise of the empirical pair correlation function) taken at finitely many pairwise distinct arguments. This includes a careful study of the corresponding asymptotic covariances. Our multivariate CLTs will be proved by the method of moments (as used also in Jolivet $[11,12]$ ) which consists in showing that the $k$ th cumulant of any scalar product of the normalized estimation vector with some real vector disappears asymptotically for all $k \geq 3$.

Firstly, we introduce some basic notions. Let $[M, \mathcal{M}]$ denote the measurable space of all locally finite counting measures on the $d$-dimensional Euclidean space $\mathbb{R}^{d}$ equipped with its $\sigma$-algebra $\mathcal{B}^{d}$ of Borel sets. A point process (shortly $\mathrm{PP}$ ) on $\mathbb{R}^{d}$ is defined as a measurable mapping $\Psi$ from a probability space $[\Omega, \mathcal{A}, \mathbb{P}]$ into $[M, \mathcal{M}]$. Throughout in this paper we assume that $\Psi$ is simple, i.e. $\mathbb{P}\left(\Psi(\{x\}) \leq 1\right.$ for all $\left.x \in \mathbb{R}^{d}\right)=1$, and strictly stationary. Let $\mathbb{E}$ and Var denote expectation and variance, respectively, with respect to $\mathbb{P}$. Let $P=\mathbb{P} \circ \Psi^{-1}$ denote the probability measure on $[M, \mathcal{M}]$ induced by $\Psi$ and we will briefly write $\Psi \sim P$. If $\mathbb{E} \Psi^{k}(B)<\infty$ for all bounded Borel sets $B$, then there exist the $k$ th-order factorial moment measure $\alpha^{(k)}$ and the $k$ th-order factorial cumulant measure $\gamma^{(k)}$ on $\left[\mathbb{R}^{d k}, \mathcal{B}^{d k}\right]$ defined by

$$
\alpha^{(k)}\left(\underset{j=1}{\times} B_{j}\right):=\int_{N} \sum_{x_{1}, \ldots, x_{k} \in \psi}^{*} \prod_{j=1}^{k} \mathbb{1}_{B_{j}}\left(x_{j}\right) P(\mathrm{~d} \psi)
$$

and

$$
\gamma^{(k)}\left(\underset{j=1}{\stackrel{k}{\times}} B_{j}\right):=\sum_{\ell=1}^{k}(-1)^{\ell-1}(\ell-1) ! \sum_{K_{1} \cup \ldots \cup K_{\ell}=K}^{(\ell, k)} \prod_{j=1}^{\ell} \alpha^{\left(\# K_{j}\right)}\left(\underset{k_{j} \in K_{j}}{\times} B_{k_{j}}\right)
$$

with $B_{1}, \ldots, B_{k} \in \mathcal{B}^{d}$, respectively. Here, " $x \in \psi$ " means " $x \in \mathbb{R}^{d}: \psi(\{x\})>0$ " and $\sum^{*}$ indicates summation over index tuples consisting of pairwise distinct elements. The sum $\sum^{(\ell, k)}$ is taken over all partitions of the set $K=\{1, \ldots, k\}$ into $\ell$ disjoint non-empty subsets $K_{j}$ and $\# K_{j}$ denotes the cardinality of $K_{j}$. If $\Psi \sim P$ is stationary with intensity $\lambda>0$ the $k$ th-order reduced factorial moment measure $\alpha_{\text {red }}^{(k)}$ on $\left[\mathbb{R}^{d(k-1)}, \mathcal{B}^{d(k-1)}\right]$ is implicitly defined by the disintegration

$$
\alpha^{(k)}\left(\underset{j=1}{\stackrel{x}{\times}} B_{j}\right)=\lambda \int_{B_{k}} \alpha_{\text {red }}^{(k)}\left(\underset{j=1}{\underset{x}{\times}}\left(B_{j}-x\right)\right) \mathrm{d} x,
$$

see Daley and Vere-Jones [4, p. 238] for further details. Analogously, the disintegration

$$
\gamma^{(k)}\left(\underset{j=1}{\stackrel{k}{\times}} B_{j}\right)=\lambda \int_{B_{k}} \gamma_{\text {red }}^{(k)}\left(\underset{j=1}{\underset{x}{\times}}\left(B_{j}-x\right)\right) \mathrm{d} x
$$

yields the $k$ th-order reduced factorial cumulant measure $\gamma_{\text {red }}^{(k)}$ on $\left[\mathbb{R}^{d(k-1)}, \mathcal{B}^{d(k-1)}\right]$. 
The total variation measure $\left|\gamma_{\text {red }}^{(k)}\right|$ of the signed measure $\gamma_{\text {red }}^{(k)}$ is defined by $\left|\gamma_{\text {red }}^{(k)}\right|()=$. $\left(\gamma_{\text {red }}^{(k)}\right)^{+}()+.\left(\gamma_{\text {red }}^{(k)}\right)^{-}($.$) , where the measures \left(\gamma_{\text {red }}^{(k)}\right)^{+}$and $\left(\gamma_{\text {red }}^{(k)}\right)^{-}$are the positive and the negative part, respectively, of the Jordan decomposition $\gamma_{\text {red }}^{(k)}()=.\left(\gamma_{\text {red }}^{(k)}\right)^{+}()-.\left(\gamma_{\text {red }}^{(k)}\right)^{-}($.$) .$ The total variation of $\gamma_{\text {red }}^{(k)}$ is defined by $\left.\left\|\gamma_{\text {red }}^{(k)}\right\|:=\left|\gamma_{\text {red }}^{(k)}\right|\left(\left(\mathbb{R}^{d}\right)^{k-1}\right)\right)$.

A stationary PP $\Psi \sim P$ in $\mathbb{R}^{d}$ satisfying $\mathbb{E} \Psi^{k}\left([0,1]^{d}\right)<\infty$ for some $k \geq 2$ is said to be $\mathrm{B}_{k}$-mixing if $\left\|\gamma_{\text {red }}^{(j)}\right\|<\infty$ for $j=2, \ldots, k . \Psi \sim P$ is called Brillinger-mixing or $\mathrm{B}_{\infty}$-mixing if $\Psi$ is $\mathrm{B}_{k}$-mixing for all $k \geq 2$, see Brillinger [2] (for $d=1$ ) or Karr [13, p. 372]. Heinrich [7] and Heinrich and Schmidt [9] state conditions on several classes of PPes for being $\mathrm{B}_{\infty}$-mixing.

If the $\ell$ th-order reduced factorial moment measure $\alpha_{\text {red }}^{(\ell)}$ is absolutely continuous with respect to the Lebesgue measure on $\left[\mathbb{R}^{d(\ell-1)}, \mathcal{B}^{d(\ell-1)}\right]$, then its Lebesgue density $\varrho^{(\ell)}$ is given by

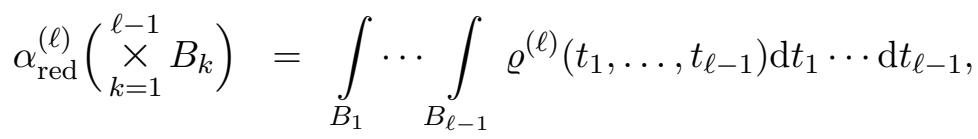

where $B_{1}, \ldots, B_{\ell-1} \in \mathcal{B}^{d}$, and is called the th-order reduced product density, henceforth abbreviated as $\ell$ th-order product density. Further we will use the abbreviation $\varrho=\varrho^{(2)}$ for the second-order product density. If the $\ell$ th-order reduced factorial cumulant measure $\gamma_{\text {red }}^{(\ell)}$ is absolutely continuous with respect to the Lebesgue measure on $\left[\mathbb{R}^{d(\ell-1)}, \mathcal{B}^{d(\ell-1)}\right]$, then its Lebesgue density $c^{(\ell)}$ is given by

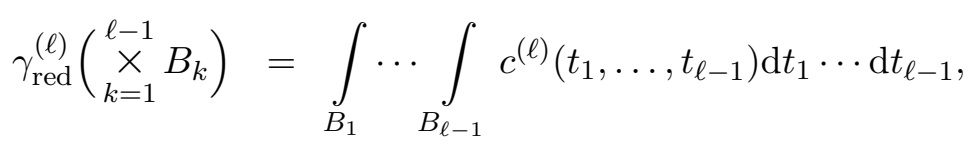

where $B_{1}, \ldots, B_{\ell-1} \in \mathcal{B}^{d}$, and is called the $\ell$ th-order reduced cumulant density, henceforth abbreviated as $\ell$ th-order cumulant density.

The isotropic analogue of the second-order product density, the pair correlation function $(\mathrm{PCF})$, is defined by

$$
g(r):=\frac{\varrho(x)}{\lambda},
$$

where $r=\|x\|, x \in \mathbb{R}^{d}$, and $\lambda$ is the intensity of the stationary PP.

The rest of the paper is organized as follows. Section 2 introduces the estimators for the product densities and the PCF. In Section 3 we derive CLTs for the empirical second-order product density. In the following two sections these results are transferred to the empirical PCF and to the empirical higher-order product densities. The appendix summarizes some facts on "indecomposable integrals" needed for the proofs of the CLTs.

\section{Empirical product densities}

In this section we will present the estimators for the $\ell$ th-order product densities $(\ell \geq 2)$ and the $\mathrm{PCF}$ and formulate some conditions needed for our asymptotic results in the next sections. 
Let $\rho(W):=\sup \left\{r \geq 0: b(x, r) \subset W, x \in \mathbb{R}^{d}\right\}$ denote the inradius of the set $W \subset \mathbb{R}^{d}$, where $b(x, r):=\left\{y \in \mathbb{R}^{d}:\|y-x\| \leq r\right\}$ is the ball with radius $r \geq 0$ centered at $x \in \mathbb{R}^{d}$. Let $|$. denote the Lebesgue measure on $\left[\mathbb{R}^{d}, \mathcal{B}^{d}\right]$ and let $\omega_{d}=|b(o, 1)|$. The following condition is needed for the precise definition of the kernel-type product density estimators.

$\operatorname{Wbk}(m)$.

(i) The sequence of observation windows $\left(W_{n}\right)$ is an increasing sequence of convex and compact sets in $\mathbb{R}^{d}$ with $\rho\left(W_{n}\right) \underset{n \rightarrow \infty}{\longrightarrow} \infty$,

(ii) the sequence of bandwidths $\left(b_{n}\right)$ is a decreasing sequence of positive real numbers satisfying $b_{n} \underset{n \rightarrow \infty}{\longrightarrow} 0$ and $b_{n}^{m}\left|W_{n}\right| \underset{n \rightarrow \infty}{\longrightarrow} \infty$, and

(iii) the kernel function $k_{m}: \mathbb{R}^{m} \rightarrow \mathbb{R}$ is bounded with bounded support, symmetric (i.e., $k_{m}(x)=k_{m}(-x)$ for every $\left.x \in \mathbb{R}^{m}\right)$, and satisfies $\int_{\mathbb{R}^{m}} k_{m}(x) \mathrm{d} x=1$.

Later on we will use the abbreviation $\left\|k_{m}\right\|:=\left(\int_{\mathbb{R}^{m}} k_{m}(x) \mathrm{d} x\right)^{1 / 2}$. The following definition of a kernel-type estimator for the second-order product density goes back to Krickeberg [14]. The speed of $\mathcal{L}_{p}$-convergence of this estimator has been studied in Jolivet [12].

Definition. Let $\left(W_{n}\right),\left(b_{n}\right)$, and $k_{d}$ satisfy Condition $\mathbf{W b k}(\boldsymbol{d})$. Let the $P P \Psi \sim P$ in $\mathbb{R}^{d}$ be stationary and assume its product density $\varrho$ to exist. Then we define

$$
\hat{\varrho}_{n}(t):=\frac{1}{b_{n}^{d}\left|W_{n}\right|} \sum_{x_{1}, x_{2} \in \Psi}^{*} \mathbb{1}_{W_{n}}\left(x_{1}\right) k_{d}\left(\frac{x_{2}-x_{1}-t}{b_{n}}\right)
$$

as an estimator for $\lambda \varrho(t)$ for $t \in \mathbb{R}^{d}$.

The above definition is generalized for higher-order product density estimators as follows.

Definition. Let $\left(W_{n}\right),\left(b_{n}\right)$, and $k_{d(\ell-1)}$ satisfy Condition $\mathbf{W b k}(\boldsymbol{d}(\boldsymbol{\ell}-\mathbf{1}))$. Let the PP $\Psi \sim P$ in $\mathbb{R}^{d}$ be stationary and assume its lth-order product density $\varrho$ to exist. Then we define

$\hat{\varrho}_{n}^{(\ell)}\left(t_{1}, \ldots, t_{\ell-1}\right):=\frac{1}{b_{n}^{d(\ell-1)}\left|W_{n}\right|} \sum_{x_{1}, \ldots, x_{\ell} \in \Psi}^{*} \mathbb{1}_{W_{n}}\left(x_{1}\right) k_{d(\ell-1)}\left(\frac{x_{2}-x_{1}-t_{1}}{b_{n}}, \ldots, \frac{x_{\ell}-x_{1}-t_{\ell-1}}{b_{n}}\right)$

as an estimator for $\lambda \varrho^{(\ell)}(t)$ for $t=\left(t_{1}, \ldots, t_{\ell-1}\right) \in \mathbb{R}^{d(\ell-1)}$.

Furthermore we consider the following kernel estimator for the PCF.

Definition. Let $\left(W_{n}\right),\left(b_{n}\right)$, and $k_{1}$ satisfy Condition $\mathbf{W b k}(\mathbf{1})$. Let the $P P \Psi \sim P$ in $\mathbb{R}^{d}$ be stationary and assume its PCF $g$ to exist. Then we define

$$
\hat{g}_{n}(r):=\frac{1}{b_{n}\left|W_{n}\right| d \omega_{d}} \sum_{x_{1}, x_{2} \in \Psi}^{*} \frac{\mathbb{1}_{W_{n}}\left(x_{1}\right)}{\left\|x_{2}-x_{1}\right\|^{d-1}} k_{1}\left(\frac{\left\|x_{2}-x_{1}\right\|-r}{b_{n}}\right)
$$

as an estimator for $\lambda^{2} g(r)$ for $r \in[0, \infty)$. 
For a discussion of this and slightly modified estimators for the PCF with regard to bias and variance see Stoyan and Stoyan [17].

In the following conditions we have $\ell \geq 2, p \geq 3$, and $s, t, s_{1}, \ldots, s_{\ell-1}, t_{1}, \ldots, t_{\ell-1} \in \mathbb{R}^{d}$.

$\gamma\left(\left(s_{i}\right)_{i=1}^{\ell-1}, p\right)$. The total variation measures $\left|\gamma_{\text {red }}^{(k)}\right|, k=\ell+1, \ldots, 2(p-1)$, satisfy

$$
\limsup _{\varepsilon \downarrow 0} \varepsilon^{-d(\ell-1)}\left|\gamma_{\text {red }}^{(k)}\right|\left(\underset{i=1}{\ell-1} \underset{\times}{\times} b\left(s_{i}, \varepsilon\right) \times \mathbb{R}^{d(k-\ell)}\right)<\infty .
$$

We write that Condition $\gamma\left(\left(s_{i}\right)_{i=1}^{\ell-1}, \infty\right)$ is satisfied if Condition $\gamma\left(\left(s_{i}\right)_{i=1}^{\ell-1}, p\right)$ is satisfied for all $p \geq 3$.

$\mathbf{c}(s, \boldsymbol{t})$. The third-order and fourth-order cumulant densities $c^{(3)}$ and $c^{(4)}$ satisfy

$\sup _{u \in b(s, \varepsilon) \cup b(-s, \varepsilon), v \in b(t, \varepsilon) \cup b(-t, \varepsilon)}\left|c^{(3)}(u, v)\right|<\infty \quad$ and $\sup _{u \in b(s, \varepsilon), v \in b(t, \varepsilon)} \int_{\mathbb{R}^{d}}\left|c^{(4)}(u, w, v+w)\right| \mathrm{d} w<\infty$ for some $\varepsilon>0$.

$\mathbf{c}_{\ell}\left(\left(s_{i}, \boldsymbol{t}_{\boldsymbol{i}}\right)_{\boldsymbol{i}=1}^{\ell-1}\right)$. The cumulant densities up to order $2 \ell$ satisfy

$$
\sup _{x_{1}, \ldots, x_{j-1} \in \bigcup_{i=1}^{\ell-1} b\left(s_{i}, \varepsilon\right) \cup b\left(-s_{i}, \varepsilon\right) \cup b\left(t_{i}, \varepsilon\right) \cup b\left(-t_{i}, \varepsilon\right)}\left|c^{(j)}\left(x_{1}, \ldots, x_{j-1}\right)\right|<\infty
$$

for $j=\ell+1, \ldots, 2 \ell-1$, and

$$
\sup _{\substack{x_{i} \in b\left(s_{i}, \varepsilon\right), y_{i} \in b\left(t_{i}, \varepsilon\right), i=1, \ldots, \ell-1}} \int_{\mathbb{R}^{d}}\left|c^{(2 \ell)}\left(x_{1}, \ldots, x_{\ell-1}, z+y_{1}, z+y_{2}, \ldots, z+y_{\ell-1}, z\right)\right| \mathrm{d} z<\infty
$$

for some $\varepsilon>0$.

\section{Central limit theorems for the empirical second-order product density}

After presenting asymptotic representations of the mean and the variance of the second-order product density estimator under mild mixing conditions we derive CLTs for this estimator in the setting of $\mathrm{B}_{\infty}$-mixing PPes. In this section we write $\int$ for $\int_{\mathbb{R}^{d}}$.

\subsection{Asymptotic representation of the mean and the variance}

In this section we derive asymptotic representations for the mean and the variance of the estimator for the second-order product density under mild mixing conditions. Similar results for $\beta$-mixing PPes can be found in Heinrich and Liebscher [8].

The second-order product density $\varrho$ is said to be Lipschitz-continuous in $t \in \mathbb{R}^{d}$ if there exists an $L<\infty$ (which may depend on $t$ ) such that $|\varrho(t)-\varrho(y)| \leq L\|t-y\|$ for all $y \in b(t, \varepsilon)$ and some $\varepsilon>0$. 
Proposition 3.1. Let $\Psi \sim P$ be a stationary $P P$ in $\mathbb{R}^{d}$ with intensity $\lambda$ and second-order product density $\varrho$. Let $\left(W_{n}\right),\left(b_{n}\right)$, and $k_{d}$ satisfy Condition $\mathbf{W b k}(\boldsymbol{d})$. Then we have

$$
\lim _{n \rightarrow \infty} \mathbb{E} \hat{\varrho}_{n}(t)=\lambda \varrho(t)
$$

in every point of continuity $t \in \mathbb{R}^{d}$ of $\varrho$.

In addition, let $\varrho$ be Lipschitz-continuous in $t$. Then we have

$$
\mathbb{E} \hat{\varrho}_{n}(t)=\lambda \varrho(t)+\mathcal{O}\left(b_{n}\right) \quad \text { as } n \rightarrow \infty .
$$

Proof. Due to $\mathbb{E} \hat{\varrho}_{n}(t)=\int k_{d}(y) \lambda \varrho\left(b_{n} y+t\right) \mathrm{d} y$, the continuity of $\varrho$ in $t$ and the boundedness conditions on the kernel function yield the first conjecture by Lebesgue's dominated convergence theorem.

If $\varrho$ is Lipschitz-continuous in $t$ with Lipschitz constant $L$ for some $\varepsilon>0$, we obtain the second conjecture by

$$
\left|\mathbb{E} \hat{\varrho}_{n}(t)-\lambda \varrho(t)\right| \leq b_{n} \lambda L \int|y|\left|k_{d}(y)\right| \mathrm{d} y=\mathcal{O}\left(b_{n}\right) .
$$

The following theorem gives an asymptotic representation of the covariance $\operatorname{Cov}\left(\varrho_{n}(s), \hat{\varrho}_{n}(t)\right)$ of the estimated second-order product density in two points $s, t \in \mathbb{R}^{d}$. In Heinrich [7, Theorem 5], the limit $b_{n}^{d}\left|W_{n}\right| \operatorname{Cov}\left(\varrho_{n}(s), \hat{\varrho}_{n}(t)\right)$ has already been determined, based on assumptions on the densities $p^{(2)}, p^{(3)}$ and $p^{(4)}$ of the moment measures of order two, three and four, not on assumptions on cumulant densities as we have here. Note that Heinrich [7, Theorem 5] misstates the limiting variance of the second-order product density estimator in zero. An extra factor 2 has to be added for correction.

Theorem 3.2. Let $\Psi \sim P$ be a $\mathrm{B}_{4}$-mixing $P P$ in $\mathbb{R}^{d}$ with intensity $\lambda$ and second-order product density $\varrho$. Let $\left(W_{n}\right),\left(b_{n}\right)$, and $k$ satisfy Condition $\mathbf{W b k}(\boldsymbol{d})$. Let the third-order and fourth-order cumulant densities $c^{(3)}$ and $c^{(4)}$ exist and satisfy Condition $\mathbf{c}(\boldsymbol{s}, \boldsymbol{t})$. Then we have

$$
b_{n}^{d}\left|W_{n}\right| \operatorname{Cov}\left(\hat{\varrho}_{n}(s), \hat{\varrho}_{n}(t)\right)=\left\{\begin{array}{cl}
\lambda \varrho(s)\left\|k_{d}\right\|^{2}+\mathrm{o}(1), & s= \pm t \neq o, \\
2 \lambda \varrho(o)\left\|k_{d}\right\|^{2}+\mathrm{o}(1), & s=t=o, \\
\mathcal{O}\left(b_{n}^{d}\right), & s \neq \pm t
\end{array}\right.
$$

in every point of continuity $s \in \mathbb{R}^{d}$ of $\varrho$ as $n \rightarrow \infty$.

If, in addition, the second-order product density @ is Lipschitz-continuous in s, then we have

$$
b_{n}^{d}\left|W_{n}\right| \operatorname{Cov}\left(\hat{\varrho}_{n}(s), \hat{\varrho}_{n}(t)\right)= \begin{cases}\lambda \varrho(s)\left\|k_{d}\right\|^{2}+\mathcal{O}\left(b_{n}\right), & s= \pm t \neq o, \\ 2 \lambda \varrho(o)\left\|k_{d}\right\|^{2}+\mathcal{O}\left(b_{n}\right), & s=t=o, \\ \mathcal{O}\left(b_{n}^{d}\right), & s \neq \pm t, \quad \text { as } n \rightarrow \infty .\end{cases}
$$


Proof. By straightforward calculation the term $b_{n}^{2 d}\left|W_{n}\right|^{2} \operatorname{Cov}\left(\varrho_{n}(s), \varrho_{n}(t)\right)$ can be rewritten as

$$
\begin{aligned}
& \int_{\left(\mathbb{R}^{d}\right)^{2}} \mathbb{1}_{W_{n}}(x) k_{d}\left(\frac{y-x-s}{b_{n}}\right)\left[k_{d}\left(\frac{y-x-t}{b_{n}}\right)+\mathbb{1}_{W_{n}}(y) k_{d}\left(\frac{x-y-t}{b_{n}}\right)\right] \alpha^{(2)}(\mathrm{d}(x, y)) \\
& +\int_{\left(\mathbb{R}^{d}\right)^{3}} \mathbb{1}_{W_{n}}(x) k_{d}\left(\frac{y-x-s}{b_{n}}\right) k_{d}\left(\frac{z-x-t}{b_{n}}\right) \alpha^{(3)}(\mathrm{d}(x, y, z)) \\
& +\int_{\left(\mathbb{R}^{d}\right)^{3}} \mathbb{1}_{W_{n}}(x) \mathbb{1}_{W_{n}}(y) k_{d}\left(\frac{y-x-s}{b_{n}}\right) k_{d}\left(\frac{z-y-t}{b_{n}}\right) \alpha^{(3)}(\mathrm{d}(x, y, z)) \\
& +\int_{\left(\mathbb{R}^{d}\right)^{3}} \mathbb{1}_{W_{n}}(x) \mathbb{1}_{W_{n}}(z) k_{d}\left(\frac{y-x-s}{b_{n}}\right) k_{d}\left(\frac{x-z-t}{b_{n}}\right) \alpha^{(3)}(\mathrm{d}(x, y, z)) \\
& +\int_{\left(\mathbb{R}^{d}\right)^{3}} \mathbb{1}_{W_{n}}(x) \mathbb{1}_{W_{n}}(z) k_{d}\left(\frac{y-x-s}{b_{n}}\right) k_{d}\left(\frac{y-z-t}{b_{n}}\right) \alpha^{(3)}(\mathrm{d}(x, y, z)) \\
& +\int_{\left(\mathbb{R}^{d}\right)^{4}} \mathbb{1}_{W_{n}}(x) \mathbb{1}_{W_{n}}(z) k_{d}\left(\frac{y-x-s}{b_{n}}\right) k_{d}\left(\frac{v-z-t}{b_{n}}\right) \\
& {\left[\alpha^{(4)}(\mathrm{d}(x, y, z, v))-\alpha^{(2)}(\mathrm{d}(x, y)) \alpha^{(2)}(\mathrm{d}(z, v))\right],}
\end{aligned}
$$

where the signed measure $\alpha^{(4)}(\mathrm{d}(x, y, z, v))-\alpha^{(2)}(\mathrm{d}(x, y)) \alpha^{(2)}(\mathrm{d}(z, v))$ can be written as

$$
\begin{aligned}
& \gamma^{(4)}(\mathrm{d}(x, y, z, v))+\gamma^{(1)}(\mathrm{d} x) \gamma^{(3)}(\mathrm{d}(y, z, v))+\gamma^{(1)}(\mathrm{d} y) \gamma^{(3)}(\mathrm{d}(x, z, v)) \\
& +\gamma^{(1)}(\mathrm{d} z) \gamma^{(3)}(\mathrm{d}(x, y, v))+\gamma^{(1)}(\mathrm{d} v) \gamma^{(3)}(\mathrm{d}(x, y, z))+\gamma^{(2)}(\mathrm{d}(x, z)) \gamma^{(2)}(\mathrm{d}(y, v)) \\
& +\gamma^{(2)}(\mathrm{d}(x, v)) \gamma^{(2)}(\mathrm{d}(y, z))+\gamma^{(2)}(\mathrm{d}(x, z)) \gamma^{(1)}(\mathrm{d} y) \gamma^{(1)}(\mathrm{d} v)+\gamma^{(2)}(\mathrm{d}(x, v)) \gamma^{(1)}(\mathrm{d} y) \gamma^{(1)}(\mathrm{d} z) \\
& +\gamma^{(2)}(\mathrm{d}(y, z)) \gamma^{(1)}(\mathrm{d} x) \gamma^{(1)}(\mathrm{d} v)+\gamma^{(2)}(\mathrm{d}(y, v)) \gamma^{(1)}(\mathrm{d} x) \gamma^{(1)}(\mathrm{d} z),
\end{aligned}
$$

see also equation (4.17) in Heinrich [7]. When multiplied by $b_{n}^{d}\left|W_{n}\right|$, only the first integral does not converge to zero for $s \neq t$. We have

$$
\begin{aligned}
& \frac{1}{b_{n}^{d}\left|W_{n}\right|} \int_{\left(\mathbb{R}^{d}\right)^{2}} \mathbb{1}_{W_{n}}(x) k_{d}\left(\frac{y-x-s}{b_{n}}\right) k_{d}\left(\frac{y-x-t}{b_{n}}\right) \alpha^{(2)}(\mathrm{d}(x, y)) \\
& =\lambda \varrho(s) \int k_{d}(y) k_{d}\left(y+\frac{s-t}{b_{n}}\right) \mathrm{d} y+\lambda \int k_{d}(y) k_{d}\left(y+\frac{s-t}{b_{n}}\right)\left(\varrho\left(b_{n} y+s\right)-\varrho(s)\right) \mathrm{d} y \\
& =\left\{\begin{array}{ll}
\lambda \varrho(s) \int k_{d}^{2}(x) \mathrm{d} x+o(1) & \text { for } s=t \\
0 & \text { for } s \neq t
\end{array} \text { as } n \rightarrow \infty\right.
\end{aligned}
$$

by continuity of $\varrho$ in $s$ and the bounded support of the kernel function $k_{d}$. Note that in the case $s \neq t$ the error term equals zero for sufficiently large $n$ since $k_{d}$ has bounded support. If we use $\left|\varrho\left(b_{n} y+s\right)-\varrho(s)\right| \leq L b_{n}\|y\|$ by the additional assumption of Lipschitz-continuity in $s$, we get $\lambda \varrho(s) \int k_{d}^{2}(x) \mathrm{d} x+\mathcal{O}\left(b_{n}\right)$ for the above integral for $s=t$ as $n \rightarrow \infty$. 
Furthermore, multiplying by $b_{n}^{d}\left|W_{n}\right|$ we get

$$
\begin{aligned}
& \frac{1}{b_{n}^{d}\left|W_{n}\right|} \int_{\left(\mathbb{R}^{d}\right)^{2}} \mathbb{1}_{W_{n}}(x) \mathbb{1}_{W_{n}}(y) k_{d}\left(\frac{y-x-s}{b_{n}}\right) k_{d}\left(\frac{x-y-t}{b_{n}}\right) \alpha^{(2)}(\mathrm{d}(x, y)) \\
& =\lambda \int \frac{\left|W_{n} \cap\left(W_{n}-b_{n} y-s\right)\right|}{\left|W_{n}\right|} k_{d}(y) k_{d}\left(y+\frac{s+t}{b_{n}}\right) \varrho\left(b_{n} y+s\right) \mathrm{d} y \\
& = \begin{cases}\lambda \varrho(s) \int k_{d}^{2}(x) \mathrm{d} x+o(1) & \text { for } s=-t \\
0 & \text { for } s \neq-t\end{cases}
\end{aligned}
$$

in every point of continuity $s \in \mathbb{R}^{d}$. As before, in the case $s \neq-t$ the error term equals zero for sufficiently large $n$ since $k_{d}$ has bounded support. The rate of convergence for this integral under the assumption of Lipschitz-continuity is the same as for the first integral.

We will now show that all the other integrals are of order $\mathcal{O}\left(b_{n}^{d}\right)$ when multiplied with $b_{n}^{d}\left|W_{n}\right|$. For the first integral with respect to the third-order factorial moment measure $\alpha^{(3)}$ we have

$$
\begin{aligned}
& \frac{1}{b_{n}^{2 d}\left|W_{n}\right|^{2}} \int_{\left(\mathbb{R}^{d}\right)^{3}} \mathbb{1}_{W_{n}}(x) k_{d}\left(\frac{y-x-s}{b_{n}}\right) k_{d}\left(\frac{z-x-t}{b_{n}}\right) \alpha^{(3)}(\mathrm{d}(x, y, z)) \\
& =\frac{1}{b_{n}^{2 d}\left|W_{n}\right|^{2}} \int_{\left(\mathbb{R}^{d}\right)^{3}} \mathbb{1}_{W_{n}}(x) k_{d}\left(\frac{y-x-s}{b_{n}}\right) k_{d}\left(\frac{z-x-t}{b_{n}}\right)\left[\gamma^{(3)}(\mathrm{d}(x, y, z))\right. \\
& \left.\quad+\lambda \mathrm{d} x \gamma^{(2)}(\mathrm{d}(y, z))+\lambda \mathrm{d} y \gamma^{(2)}(\mathrm{d}(x, z))+\lambda \mathrm{d} z \gamma^{(2)}(\mathrm{d}(x, y))+\lambda^{3} \mathrm{~d} x \mathrm{~d} y \mathrm{~d} z\right] .
\end{aligned}
$$

Multiplying with $b_{n}^{d}\left|W_{n}\right|$ we find

$$
\begin{aligned}
& \frac{1}{b_{n}^{d}\left|W_{n}\right|} \int_{\left(\mathbb{R}^{d}\right)^{3}} \mathbb{1}_{W_{n}}(x) k_{d}\left(\frac{y-s}{b_{n}}\right) k_{d}\left(\frac{z-t}{b_{n}}\right) \gamma_{\text {red }}^{(3)}(\mathrm{d}(y, z)) \mathrm{d} x \\
& =b_{n}^{d} \lambda \int_{\left(\mathbb{R}^{d}\right)^{2}} k_{d}(y) k_{d}(z) c^{(3)}\left(b_{n} y+s, b_{n} z+t\right) \mathrm{d} y \mathrm{~d} z
\end{aligned}
$$

which is of order $\mathcal{O}\left(b_{n}^{d}\right)$ by our assumption on the third-order cumulant density $c^{(3)}$. Analogously, one may show the other integrals with respect to the third-order factorial moment measure $\alpha^{(3)}$ to be of order $\mathcal{O}\left(b_{n}^{d}\right)$.

The integrals with respect to the factorial cumulant measures can be treated analogously. For the integral with respect to $\gamma^{(4)}$, multiplied with $b_{n}^{d}\left|W_{n}\right|$, we get

$$
\begin{aligned}
& \frac{1}{b_{n}^{d}\left|W_{n}\right|} \int_{\left(\mathbb{R}^{d}\right)^{4}} \mathbb{1}_{W_{n}}(x) \mathbb{1}_{W_{n}}(z) k_{d}\left(\frac{y-x-s}{b_{n}}\right) k_{d}\left(\frac{v-z-t}{b_{n}}\right) \gamma^{(4)}(\mathrm{d}(x, y, z, v)) \\
& =b_{n}^{d} \lambda \int_{\left(\mathbb{R}^{d}\right)^{4}} \frac{\left|W_{n} \cap\left(W_{n}-z\right)\right|}{\left|W_{n}\right|} k_{d}(y) k_{d}(v) c^{(4)}\left(b_{n} y+s, z, b_{n} v+z+t\right) \mathrm{d} y \mathrm{~d} z \mathrm{~d} v
\end{aligned}
$$

which is of asymptotic order $\mathcal{O}\left(b_{n}^{d}\right)$ due to the assumption on the fourth-order cumulant density $c^{(4)}$. Similar arguments show that the other integrals are of asymptotic order $\mathcal{O}\left(b_{n}^{d}\right)$, too. 


\subsection{Central limit theorems}

For Poisson cluster processes Heinrich [7] proves a CLT for the sequence

$$
\Delta_{n}(\varrho, t):=\sqrt{b_{n}^{d}\left|W_{n}\right|}\left(\hat{\varrho}_{n}(t)-\mathbb{E} \hat{\varrho}_{n}(t)\right), \quad t \in \mathbb{R}^{d},
$$

by using methods for $m$-dependent point fields. However, these methods cannot be applied in the setting of $\mathrm{B}_{\infty}$-mixing PPes. In the latter case we will prove a CLT by showing the $k$-th cumulants of the above-mentioned sequence to converge to zero for $k \geq 3$.

Jolivet [12] determines the order of the $k$ th cumulant of the $\ell$ th-order product density $\varrho_{n}^{(\ell)}$, $\ell \geq 2$, by using methods by Leonov and Shiryaev [15] and Jolivet [11]. However, Jolivet [12] only investigates the terms of highest order and does not take into account that some assumptions on the cumulant densities and the total variation measures have to be made in order to prove that the lower-order terms converge to zero.

In the following $\underset{n \rightarrow \infty}{\mathrm{d}}$ denotes convergence in distribution, while $\chi_{q}^{2}$ and $\mathcal{N}\left(0_{q}, \Sigma_{q}\right)$ denote the $\chi^{2}$-distribution with $q$ degrees of freedom and the $q$-variate normal distribution with zero mean vector $0_{q} \in \mathbb{R}^{q}$ and positive-semidefinite $q \times q$-covariance matrix $\Sigma_{q}$, respectively.

Theorem 3.3. Let $\Psi \sim P$ be a $\mathrm{B}_{\infty}$-mixing $P P$ in $\mathbb{R}^{d}$ with intensity $\lambda$ and second-order product density $\varrho$. Let $\left(W_{n}\right),\left(b_{n}\right)$, and $k_{d}$ satisfy Condition $\mathbf{W b k}(\boldsymbol{d})$. Let the q-tuple $\left(u_{1}, \ldots, u_{q}\right) \in\left(\mathbb{R}^{d} \backslash\{o\}\right)^{q}$ be chosen such that $u_{i} \neq \pm u_{j}$ for $i \neq j$, and let $u_{i}$ be a point of continuity of $\varrho$ for every $i=1, \ldots, q$. Furthermore, let Condition $\gamma\left(\boldsymbol{u}_{\boldsymbol{i}}, \infty\right)$ be satisfied and let the third- and fourth-order cumulant densities exist and satisfy Condition $\mathbf{c}\left(\boldsymbol{u}_{\boldsymbol{i}}, \boldsymbol{u}_{\boldsymbol{j}}\right)$ for all $i, j=1, \ldots, q$.

Then we have $\left(\Delta_{n}\left(\varrho, u_{i}\right)\right)_{i=1}^{q} \underset{n \rightarrow \infty}{\stackrel{\mathrm{d}}{\longrightarrow}} \mathcal{N}\left(0_{q}, \Sigma_{q}\right)$, where the covariance matrix $\Sigma_{q}=\left(\sigma_{i j}\right)_{i, j=1}^{q}$ is given by $\sigma_{i i}:=\lambda \varrho\left(u_{i}\right)\left\|k_{d}\right\|^{2}$ for $i=1, \ldots, q$, and $\sigma_{i j}=0$ for $i \neq j$. Furthermore we have

$$
\sum_{i=1}^{q} \frac{\left(\Delta_{n}\left(\varrho, u_{i}\right)\right)^{2}}{\sigma_{i i}} \underset{n \rightarrow \infty}{\stackrel{\mathrm{d}}{\longrightarrow}} \chi_{q}^{2} .
$$

Proof. The asymptotic covariance has already been determined in Theorem 3.2. In order to show normal convergence of $\left(\Delta_{n}\left(\varrho, u_{i}\right)\right)_{i=1}^{q}$ we use the method of Cramér-Wold and consider the linear combination $a_{1} \Delta_{n}\left(\varrho, u_{1}\right)+\ldots+a_{q} \Delta_{n}\left(\varrho, u_{q}\right)$ for an arbitrary $q$-tuple $\left(a_{1}, \ldots, a_{q}\right) \in$ $\mathbb{R}^{q}$. Asymptotic normality of this linear combination will be established by showing that its cumulants of order $k$ converge to zero for all $k \geq 3$.

Applying the lemma given in the appendix and using the notation given there the $k$ th cumulant of $a_{1} \Delta_{n}\left(\varrho, u_{1}\right)+\ldots+a_{q} \Delta_{n}\left(\varrho, u_{q}\right), k \geq 2$, satisfies

$$
\Gamma_{k}\left(a_{1} \Delta_{n}\left(\varrho, u_{1}\right)+\ldots+a_{q} \Delta_{n}\left(\varrho, u_{q}\right)\right)
$$




$$
\begin{aligned}
& =\Gamma_{k}\left(\sum_{i=1}^{q}\left(b_{n}^{d}\left|W_{n}\right|\right)^{-1 / 2} a_{i} \Psi^{(2)}\left(f_{i}\right)\right) \\
& =\left(b_{n}^{d}\left|W_{n}\right|\right)^{-k / 2} \sum_{\substack{k_{1}+\ldots+k_{q}=k \\
k_{1}, \ldots, k_{q} \geq 0}} \frac{k !}{k_{1} ! \cdot \ldots \cdot k_{q} !} a_{1}^{k_{1}} \cdot \ldots \cdot a_{q}^{k_{q}} \mu_{k_{1}, \ldots, k_{q}}^{*}
\end{aligned}
$$

with $f_{i}\left(x_{1}, x_{2}\right)=\mathbb{1}_{W_{n}}\left(x_{1}\right) k_{d}\left(\left(x_{2}-x_{1}-u_{i}\right) / b_{n}\right)$ for $i=1, \ldots, q$.

Now we have to determine the growth rate in $n$ of $\mu_{k_{1}, \ldots, k_{q}}^{*}$ for $k_{1}, \ldots, k_{q} \geq 0$ with $\sum_{i=1}^{q} k_{i}=k$. Since $\mu_{k_{1}, \ldots, k_{q}}^{*}$ consists only of indecomposable integrals it can be seen by disintegration and substitution that the highest order of integrals with respect to at least two factorial cumulant measures is $\mathcal{O}\left(b_{n}^{d}\left|W_{n}\right|\right)$ due to $\left\|\gamma_{\text {red }}^{(j)}\right\|<\infty$ for $j \geq 2$ and the boundedness assumptions on the kernel function. Together with the factor $\left(b_{n}^{d}\left|W_{n}\right|\right)^{-k / 2}$ this yields the asymptotic order $\mathcal{O}\left(\left(b_{n}^{d}\left|W_{n}\right|\right)^{1-k / 2}\right)$ of these terms. For an integral taken with respect to only one factorial cumulant measure, disintegration and the finiteness of the total variations yields the asymptotic order $\mathcal{O}\left(\left|W_{n}\right|\right)$. For example, for the integral

$$
\int_{\left(\mathbb{R}^{d}\right)^{k}} \mathbb{1}_{W_{n}}\left(x_{1}\right) \prod_{i=2}^{k} k_{d}\left(\frac{x_{i}-x_{1}-u_{1}}{b_{n}}\right) \gamma^{(k)}\left(\mathrm{d}\left(x_{1}, \ldots, x_{k}\right)\right)
$$

we get

$$
\left|W_{n}\right| \lambda \int_{\left(\mathbb{R}^{d}\right)^{k-1}} \prod_{i=2}^{k} k_{d}\left(\frac{x_{i}-u_{1}}{b_{n}}\right) \gamma_{\mathrm{red}}^{(k)}\left(\mathrm{d}\left(x_{2}, \ldots, x_{k}\right)\right)
$$

by disintegration. The asymptotic order $\mathcal{O}\left(\left|W_{n}\right|\right)$ of this term is insufficient for our purposes. Using the boundedness conditions on the kernel function $k_{d}$, we achieve the upper bound $C \cdot\left|W_{n}\right| \int_{\left(\mathbb{R}^{d}\right)^{k-1}} \mathbb{1}_{b\left(u_{1}, b_{n} R\right)}\left(x_{2}\right)\left|\gamma_{\text {red }}^{(k)}\right|\left(\mathrm{d}\left(x_{2}, \ldots, x_{k}\right)\right)=C \cdot\left|W_{n}\right|\left|\gamma_{\text {red }}^{(k)}\right|\left(b\left(u_{1}, b_{n} R\right) \times\left(\mathbb{R}^{d}\right)^{k-2}\right)$

for the absolute value of the term (1), where $C$ and $R$ do not depend on $n$. Now Condition $\gamma\left(\boldsymbol{u}_{1}, \infty\right)$ yields the asymptotic order $\mathcal{O}\left(b_{n}^{d}\left|W_{n}\right|\right)$ of this term. For an integral taken with respect to $\gamma^{(2)}$ the continuity of the second-order product density in $u_{i}, i=1, \ldots, q$, (which implies the continuity of $c^{(2)}$ in these points due to $\left.\varrho()=.c^{(2)}()+.\lambda\right)$ and Lebesgue's dominated convergence theorem again yield the asymptotic order $\mathcal{O}\left(b_{n}^{d}\left|W_{n}\right|\right)$.

Altogether we have

$$
\Gamma_{k}\left(a_{1} \Delta_{n}\left(\varrho, u_{1}\right)+\ldots+a_{q} \Delta_{n}\left(\varrho, u_{q}\right)\right)=\mathcal{O}\left(\left(b_{n}^{d}\left|W_{n}\right|\right)^{1-k / 2}\right)
$$

as $n \rightarrow \infty$. Hence the cumulants of order $k \geq 3$ converge to zero as $n \rightarrow \infty$ which implies the claimed normal convergence. The weak convergence of

$$
\sum_{i=1}^{q} \frac{\left(\Delta_{n}\left(\varrho, u_{i}\right)\right)^{2}}{\sigma_{i i}}
$$

to a $\chi^{2}$-distributed random variable with $q$ degrees of freedom follows immediately by the Continuous Mapping Theorem. 
Remark 3.4. Theorem 3.3 still holds if Condition $\gamma\left(\boldsymbol{u}_{\boldsymbol{i}}, \infty\right)$ is replaced by $\gamma\left(\boldsymbol{u}_{\boldsymbol{i}}, \boldsymbol{p}\right)$ and the additional assumption $\left(b_{n}^{d}\right)^{p}\left|W_{n}\right|^{p-2} \underset{n \rightarrow \infty}{\longrightarrow} \infty$ for some integer $p \geq 3$. Condition $\gamma\left(\boldsymbol{u}_{\boldsymbol{i}}, \boldsymbol{p}\right)$ is relevant only for the total variation measures up to order $2(p-1)$. This weakening of Condition $\gamma\left(\boldsymbol{u}_{\boldsymbol{i}}, \infty\right)$ is compensated by the slightly stronger assumption on the bandwidth $b_{n}$.

We will now use the asymptotic representation of the mean in Proposition 3.1 to replace the centralization $\mathbb{E} \hat{\varrho}_{n}($.$) by \lambda \varrho($.$) , and apply a variance-stabilizing transformation.$

Corollary 3.5. Let $\Psi \sim P$ be a $\mathrm{B}_{\infty}$-mixing $P P$ in $\mathbb{R}^{d}$ with intensity $\lambda$ and second-order product density $\varrho$. Let $\left(W_{n}\right),\left(b_{n}\right)$, and $k_{d}$ satisfy Condition $\mathbf{W b k}(\boldsymbol{d})$ and $b_{n}^{d+2}\left|W_{n}\right| \underset{n \rightarrow \infty}{\longrightarrow} 0$. Let the $q$-tuple $\left(u_{1}, \ldots, u_{q}\right) \in\left(\mathbb{R}^{d} \backslash\{o\}\right)^{q}$ be chosen such that $u_{i} \neq \pm u_{j}$ for $i \neq j$, and let $\varrho$ be Lipschitz-continuous in $u_{i}$ for all $i=1, \ldots, q$. Let Condition $\gamma\left(\boldsymbol{u}_{\boldsymbol{i}}, \boldsymbol{\infty}\right)$ be satisfied and let the third- and fourth-order cumulant densities exist and satisfy Condition $\mathbf{c}\left(\boldsymbol{u}_{\boldsymbol{i}}, \boldsymbol{u}_{\boldsymbol{j}}\right)$ for all $i, j=1, \ldots, q$.

Then we have

$$
\left(\frac{2 \sqrt{b_{n}^{d}\left|W_{n}\right|}}{\left\|k_{d}\right\|}\left(\sqrt{\varrho_{n}\left(u_{i}\right)}-\sqrt{\lambda \varrho\left(u_{i}\right)}\right)\right)_{i=1}^{q} \underset{n \rightarrow \infty}{\stackrel{\mathrm{d}}{\longrightarrow}} \mathcal{N}\left(0_{q}, I_{q}\right),
$$

where $I_{q}$ is the $q \times q$-identity matrix. Furthermore we have

$$
\frac{4 b_{n}^{d}\left|W_{n}\right|}{\left\|k_{d}\right\|^{2}} \sum_{i=1}^{q}\left(\sqrt{\varrho_{n}\left(u_{i}\right)}-\sqrt{\lambda \varrho\left(u_{i}\right)}\right)^{2} \underset{n \rightarrow \infty}{\stackrel{\mathrm{d}}{\longrightarrow}} \chi_{q}^{2} .
$$

Proof. The asymptotic normality of the sequence $\left(\left(b_{n}^{d}\left|W_{n}\right|\right)^{1 / 2}\left(\hat{\varrho}_{n}\left(u_{i}\right)-\lambda \varrho\left(u_{i}\right)\right)\right)_{i=1}^{q}$ is an immediate consequence of Theorem 3.3, the second part of Proposition 3.1, and $b_{n}^{d+2}\left|W_{n}\right| \underset{n \rightarrow \infty}{\longrightarrow}$ 0 . The claim is established based on the weak consistency of the estimated product density and on variance-stabilization by a square-root transformation.

Corollary 3.5 can be formulated according to the modified assumptions in Remark 3.4. Note that due to the condition $b_{n}^{2+d}\left|W_{n}\right| \underset{n \rightarrow \infty}{\longrightarrow} 0$ in Corollary 3.5 the integer $p \geq 3$ must be chosen large enough such that the condition $\left(b_{n}^{d}\right)^{p}\left|W_{n}\right|^{p-2} \underset{n \rightarrow \infty}{\longrightarrow} \infty$ in Remark 3.4 can be met.

\section{Central limit theorems for the empirical pair correlation function}

All results given in Section 3 carry over to the estimator for the PCF. In this section we assume that the $\mathrm{PP}$ is both $\mathrm{B}_{\infty}$-mixing and isotropic. We formulate the main results in Section 3.2 for the sequence

$$
\Delta_{n}(g, r):=\sqrt{b_{n}\left|W_{n}\right|}\left(\hat{g}_{n}(r)-\mathbb{E} \hat{g}_{n}(r)\right), \quad r \geq 0 .
$$

Proofs are analogous to those given for the empirical second-order product density and are therefore widely omitted. In this section we write $\int$ for $\int_{\mathbb{R}}$. 
Theorem 4.1. Let $\Psi \sim P$ be a $\mathrm{B}_{\infty}$-mixing, isotropic $P P$ in $\mathbb{R}^{d}$ with intensity $\lambda$ and $P C F$ g. Let $\left(W_{n}\right),\left(b_{n}\right)$, and $k_{1}$ satisfy Condition $\mathbf{W b k}(\mathbf{1})$. Let the $q$-tuple $\left(r_{1}, \ldots, r_{q}\right) \in(0, \infty)^{q}$ be chosen such that $r_{i} \neq r_{j}$ for $i \neq j$, and let $r_{i}$ be a point of continuity of $g$ for every $i=1, \ldots, q$. Furthermore, let Condition $\gamma(\boldsymbol{u}, \infty)$ be satisfied for all $u \in \cup_{i=1}^{q} \partial b\left(o, r_{i}\right)$, and let the third- and fourth-order cumulant densities exist and satisfy Condition $\mathbf{c}(\boldsymbol{u}, \boldsymbol{v})$ for all $u, v \in \cup_{i=1}^{q} \partial b\left(o, r_{i}\right)$.

Then we have $\left(\Delta_{n}\left(g, r_{i}\right)\right)_{i=1}^{q} \underset{n \rightarrow \infty}{\stackrel{\mathrm{d}}{\longrightarrow}} \mathcal{N}\left(0_{q}, \Sigma_{q}\right)$, where the covariance matrix $\Sigma_{q}=\left(\sigma_{i j}\right)_{i, j=1}^{q}$ is given by

$$
\sigma_{i i}:=2 \lambda^{2} \frac{g\left(r_{i}\right)}{d \omega_{d} r_{i}^{d-1}}\left\|k_{1}\right\|^{2}
$$

for $i=1, \ldots, q$, and $\sigma_{i j}=0$ for $i \neq j$. Furthermore we have

$$
\sum_{i=1}^{q} \frac{\left(\Delta_{n}\left(g, r_{i}\right)\right)^{2}}{\sigma_{i i}} \underset{n \rightarrow \infty}{\stackrel{\mathrm{d}}{\longrightarrow}} \chi_{q}^{2}
$$

Proof. We only refer to the use of Condition $\gamma(\boldsymbol{u}, \infty), u \in \cup_{i=1}^{q} \partial b\left(o, r_{i}\right)$. Again, this condition is needed for integrals like

$$
\int_{\left(\mathbb{R}^{d}\right)^{k}} \mathbb{1}_{W_{n}}\left(x_{1}\right) k_{1}\left(\frac{\left\|x_{2}-x_{1}\right\|-r_{1}}{b_{n}}\right) \prod(\ldots) \gamma^{(k)}\left(\mathrm{d}\left(x_{1}, \ldots, x_{k}\right)\right),
$$

where the product $\prod(\ldots)$ contains only functions $\mathbb{1}_{W_{n}}\left(x_{j}\right)$ and $k_{1}\left(\frac{\left\|x_{m}-x_{j}\right\|-r_{i}}{b_{n}}\right)$, where $j, m \in$ $\{2, \ldots, k\}$ with $j \neq m$ and $i=2, \ldots, q$. By disintegration and by using the boundedness conditions on the kernel function $k_{1}$, we achieve the upper bound

$$
\begin{aligned}
C & \cdot\left|W_{n}\right| \int_{\left(\mathbb{R}^{d}\right)^{k-1}} \mathbb{1}_{\left[-b_{n} R, b_{n} R\right]+r_{1}}\left(\left\|x_{2}\right\|\right)\left|\gamma_{\text {red }}^{(k)}\right|\left(\mathrm{d}\left(x_{2}, \ldots, x_{k}\right)\right) \\
& =C \cdot\left|W_{n}\right|\left|\gamma_{\text {red }}^{(k)}\right|\left(\left(b\left(o, r_{1}+b_{n} R\right) \backslash b\left(o, r_{1}-b_{n} R\right)\right) \times\left(\mathbb{R}^{d}\right)^{k-2}\right)
\end{aligned}
$$

for the absolute value of the term (2), where $C$ and $R$ are constants. Note that there are $N=\mathcal{O}\left(\varepsilon^{-(d-1)}\right)$ points $u_{1}, \ldots, u_{N} \in \partial b\left(o, r_{1}\right)$ such that $\bigcup_{i=1}^{N} b\left(u_{i}, 2 \varepsilon\right) \supseteq b\left(o, r_{1}+b_{n} R\right) \backslash$ $b\left(o, r_{1}-b_{n} R\right)$. Therefore we can use Condition $\gamma(\boldsymbol{u}, \infty)$ for all $u \in \partial b\left(o, r_{1}\right)$ in order to achieve the asymptotic order $\mathcal{O}\left(b_{n}\left|W_{n}\right|\right)$ of the term above. Other integrals of this kind are treated in the same way.

Like in Remark 3.4, Condition $\gamma(\boldsymbol{u}, \infty)$ in the above theorem can be replaced by Condition $\gamma(\boldsymbol{u}, \boldsymbol{p})$ and $b_{n}^{p}\left|W_{n}\right|^{p-2} \underset{n \rightarrow \infty}{\longrightarrow} \infty$ for some $p \geq 3$.

Corollary 4.2. Let $\Psi \sim P$ be a $\mathrm{B}_{\infty}$-mixing, isotropic $P P$ in $\mathbb{R}^{d}$ with intensity $\lambda$ and $P C F$ g. Let $\left(W_{n}\right),\left(b_{n}\right)$, and $k_{1}$ satisfy Condition $\mathbf{W b k}(\mathbf{1})$ and let $b_{n}^{3}\left|W_{n}\right| \underset{n \rightarrow \infty}{\longrightarrow} 0$. Let the q-tuple $\left(r_{1}, \ldots, r_{q}\right) \in(0, \infty)^{q}$ be chosen such that $r_{i} \neq r_{j}$ for $i \neq j$, and let $g$ be Lipschitz-continuous in $r_{i}$ for all $i=1, \ldots, q$. Let Condition $\gamma(\boldsymbol{u}, \infty)$ be satisfied for all $u \in \cup_{i=1}^{q} \partial b\left(o, r_{i}\right)$, and 
let the third-and fourth-order cumulant densities exist and satisfy Condition $\mathbf{c}(\boldsymbol{u}, \boldsymbol{v})$ for all $u, v \in \cup_{i=1}^{q} \partial b\left(o, r_{i}\right)$.

Then we have

$$
\left(\frac{\sqrt{2 d \omega_{d} b_{n}\left|W_{n}\right| r_{i}^{d-1}}}{\left\|k_{1}\right\|}\left(\sqrt{g\left(r_{i}\right)}-\sqrt{\lambda^{2} g\left(r_{i}\right)}\right)\right)_{i=1}^{q} \underset{n \rightarrow \infty}{\stackrel{\mathrm{d}}{\longrightarrow}} \mathcal{N}\left(0_{q}, I_{q}\right) .
$$

Furthermore we have

$$
\frac{2 d \omega_{d} b_{n}\left|W_{n}\right|}{\left\|k_{1}\right\|^{2}} \sum_{i=1}^{q} r_{i}^{d-1}\left(\sqrt{g\left(r_{i}\right)}-\sqrt{\lambda^{2} g\left(r_{i}\right)}\right)^{2} \underset{n \rightarrow \infty}{\stackrel{\mathrm{d}}{\longrightarrow}} \chi_{q}^{2} .
$$

\section{Central limit theorems for empirical higher-order product densities}

This section extends the results on the empirical second-order product density in Section 3 to product densities $\varrho^{(\ell)}$ of order $\ell \geq 2$. We consider the sequence

$$
\Delta_{n}\left(\varrho^{(\ell)}, t\right):=\sqrt{b_{n}^{(\ell-1) d}\left|W_{n}\right|}\left(\hat{\varrho}_{n}^{(\ell)}(t)-\mathbb{E} \varrho_{n}^{(\ell)}(t)\right), \quad t \in \mathbb{R}^{d(\ell-1)},
$$

for $\ell \geq 2$. Now we write $\int$ for $\int_{\mathbb{R}^{d(\ell-1)}}$.

Proposition 5.1. Let $\Psi \sim P$ be a stationary $P P$ in $\mathbb{R}^{d}$ with intensity $\lambda$ and $\ell$ th-order product density $\varrho^{(\ell)}, \ell \geq 2$. Let $\left(W_{n}\right),\left(b_{n}\right)$, and $k_{d(\ell-1)}$ satisfy Condition $\mathbf{W b k}(\boldsymbol{d}(\ell-\mathbf{1}))$. Then we have

$$
\lim _{n \rightarrow \infty} \mathbb{E} \hat{\varrho}_{n}^{(\ell)}(t)=\lambda \varrho^{(\ell)}(t)
$$

in every point of continuity $t \in \mathbb{R}^{d(\ell-1)}$ of $\varrho^{(\ell)}$.

In addition, let $\varrho^{(\ell)}$ be Lipschitz-continuous in $t$. Then we have

$$
\mathbb{E} \hat{\varrho}_{n}^{(\ell)}(t)=\lambda \varrho^{(\ell)}(t)+\mathcal{O}\left(b_{n}\right) \quad \text { as } n \rightarrow \infty .
$$

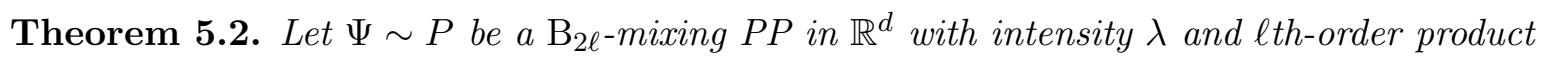
density $\varrho^{(\ell)}, \ell \geq 2$. Let $\left(W_{n}\right),\left(b_{n}\right)$, and $k_{d(\ell-1)}$ satisfy Condition $\mathbf{W b k}(\boldsymbol{d}(\boldsymbol{\ell}-\mathbf{1}))$. Let the cumulant densities up to order $2 \ell$ exist and satisfy Condition $\mathbf{c}_{\ell}\left(\left(\boldsymbol{s}_{\boldsymbol{i}}, \boldsymbol{t}_{\boldsymbol{i}}\right)_{\boldsymbol{i}=\mathbf{1}}^{\ell-\mathbf{1}}\right)$. Then we have

$$
\lim _{n \rightarrow \infty} b_{n}^{(\ell-1) d}\left|W_{n}\right| \operatorname{Cov}\left(\hat{\varrho}_{n}^{(\ell)}(s), \hat{\varrho}_{n}^{(\ell)}(t)\right)= \begin{cases}\lambda \varrho^{(\ell)}(s) \int k_{d(\ell-1)}^{2}(x) \mathrm{d} x, & s=t, \\ 0, & s \neq t,\end{cases}
$$

in every point of continuity $s=\left(s_{1}, \ldots, s_{\ell-1}\right) \in \mathbb{R}^{d(\ell-1)}$ of $\varrho^{(\ell)}$ satisfying $s_{i} \neq o$ and $s_{i} \neq \pm s_{j}$ for all $i, j \in\{1, \ldots, \ell-1\}$ with $i \neq j$. 
Theorem 5.3. Let $\Psi \sim P$ be a $\mathrm{B}_{\infty}$-mixing $P P$ in $\mathbb{R}^{d}$ with intensity $\lambda$ and th-order product density $\varrho^{(\ell)}, \ell \geq 2$. Let $\left(W_{n}\right),\left(b_{n}\right)$, and $k_{d(\ell-1)}$ satisfy Condition $\mathbf{W b k}(\boldsymbol{d}(\ell-\mathbf{1}))$. Let $u_{1}, \ldots, u_{q} \in \mathbb{R}^{d(\ell-1)}$ with $u_{i}=\left(u_{i k}\right)_{k=1}^{\ell-1} \in \mathbb{R}^{d(\ell-1)}$ be chosen such that $u_{i} \neq u_{j}$ for all $i, j \in$ $\{1, \ldots, q\}, u_{i j} \neq o$ and $u_{i j} \neq \pm u_{i k}$ for all $i \in\{1, \ldots, q\}$ and $j, k \in\{1, \ldots, \ell-1\}$ with $j \neq k$. Let $u_{i}$ be a point of continuity of $\varrho^{(\ell)}$ for every $i=1, \ldots, q$. Furthermore, let Condition $\gamma\left(\left(\boldsymbol{u}_{\boldsymbol{i}}\right)_{\boldsymbol{k}=1}^{\ell-1}, \infty\right)$ be satisfied and let the cumulant densities up to order $2 \ell$ exist and satisfy Condition $\mathbf{c}_{\ell}\left(\left(\boldsymbol{u}_{\boldsymbol{i}}, \boldsymbol{u}_{\boldsymbol{j} \boldsymbol{k}}\right)_{\boldsymbol{k}=\mathbf{\ell}}^{\ell-1}\right)$ for all $i, j=1, \ldots, q$.

Then we have $\left(\Delta_{n}\left(\varrho^{(\ell)}, u_{i}\right)\right)_{i=1}^{q} \underset{n \rightarrow \infty}{\stackrel{\mathrm{d}}{\longrightarrow}} \mathcal{N}\left(0_{q}, \Sigma_{q}\right)$, where the covariance matrix $\Sigma_{q}=\left(\sigma_{i j}\right)_{i, j=1}^{q}$ is given by

$$
\sigma_{i i}:=\lambda \varrho^{(\ell)}\left(u_{i}\right)\left\|k_{d(\ell-1)}\right\|^{2}
$$

for $i=1, \ldots, q$, and $\sigma_{i j}=0$ for $i \neq j$. Furthermore we have

$$
\sum_{i=1}^{q} \frac{\left(\Delta_{n}\left(\varrho^{(\ell)}, u_{i}\right)\right)^{2}}{\sigma_{i i}} \underset{n \rightarrow \infty}{\stackrel{\mathrm{d}}{\longrightarrow}} \chi_{q}^{2}
$$

Proof. The proof parallels the one of Theorem 3.3 for the second-order product density estimator. Again, we apply the lemma given in the appendix. Using the notation given there the $k$ th cumulant of $a_{1} \Delta_{n}\left(\varrho^{(\ell)}, u_{1}\right)+\ldots+a_{q} \Delta_{n}\left(\varrho^{(\ell)}, u_{q}\right), k \geq 2$, for an arbitrary $q$-tuple $\left(a_{1}, \ldots, a_{q}\right) \in \mathbb{R}^{q}$ satisfies

$$
\begin{aligned}
& \Gamma_{k}\left(a_{1} \Delta_{n}\left(\varrho^{(\ell)}, u_{1}\right)+\ldots+a_{q} \Delta_{n}\left(\varrho^{(\ell)}, u_{q}\right)\right) \\
& \quad=\Gamma_{k}\left(\sum_{i=1}^{q}\left(b_{n}^{(\ell-1) d}\left|W_{n}\right|\right)^{-1 / 2} a_{i} \Psi^{(\ell)}\left(f_{i}\right)\right) \\
& \quad=\left(b_{n}^{(\ell-1) d}\left|W_{n}\right|\right)^{-k / 2} \sum_{\substack{k_{1}+\ldots+k_{q}=k \\
k_{1}, \ldots, k_{q} \geq 0}} \frac{k !}{k_{1} ! \cdot \ldots \cdot k_{q} !} a_{1}^{k_{1}} \cdot \ldots \cdot a_{q}^{k_{q}} \mu_{k_{1}, \ldots, k_{q}}^{*}
\end{aligned}
$$

with $f_{i}\left(x_{1}, \ldots, x_{\ell}\right)=\mathbb{1}_{W_{n}}\left(x_{1}\right) k_{d(\ell-1)}\left(\frac{x_{2}-x_{1}-u_{i 1}}{b_{n}}, \ldots, \frac{x_{\ell}-x_{1}-u_{i(\ell-1)}}{b_{n}}\right)$ for $i=1, \ldots, q$.

Since $\mu_{k_{1}, \ldots, k_{q}}^{*}$ consists only of indecomposable integrals, the highest order of integrals with respect to at least two factorial cumulant measures is $\mathcal{O}\left(b_{n}^{(\ell-1) d}\left|W_{n}\right|\right)$ due to $\left\|\gamma_{\text {red }}^{(j)}\right\|<\infty$ for $j \geq 2$ and the boundedness assumptions on the kernel function. Together with the factor $\left(b_{n}^{(\ell-1) d}\left|W_{n}\right|\right)^{-k / 2}$ this yields the asymptotic order $\mathcal{O}\left(\left(b_{n}^{(\ell-1) d}\left|W_{n}\right|\right)^{1-k / 2}\right)$ of these terms. For an integral taken with respect to only one factorial cumulant measure like

$$
\int_{\left(\mathbb{R}^{d}\right)^{k}} \mathbb{1}_{W_{n}}\left(x_{1}\right) k_{d(\ell-1)}\left(\frac{x_{2}-x_{1}-u_{11}}{b_{n}}, \ldots, \frac{x_{\ell}-x_{1}-u_{1(\ell-1)}}{b_{n}}\right) \prod(\ldots) \gamma^{(k)}\left(\mathrm{d}\left(x_{1}, \ldots, x_{k}\right)\right),
$$

where the product $\prod(\ldots)$ contains only functions $k_{d(\ell-1)}\left(\frac{x_{m_{1}}-x_{j}-u_{i 1}}{b_{n}}, \ldots, \frac{x_{m_{\ell-1}}-x_{j}-u_{i(\ell-1)}}{b_{n}}\right)$ and $\mathbb{1}_{W_{n}}\left(x_{j}\right)$ with $i, j, m_{1}, \ldots m_{\ell-1} \neq 1$, Condition $\gamma\left(\left(\boldsymbol{u}_{\boldsymbol{i} \boldsymbol{k}}\right)_{\boldsymbol{k}=1}^{\ell-1}, \infty\right)$ is needed. By disintegration and by using the boundedness conditions on the kernel function $k_{d(\ell-1)}$ we first achieve 
the upper bound

$$
\begin{aligned}
C & \cdot\left|W_{n}\right| \int_{\left(\mathbb{R}^{d}\right)^{k-1}} \mathbb{1}_{b\left(u_{1}, b_{n} R\right)}\left(x_{2}\right) \ldots \mathbb{1}_{b\left(u_{1}, b_{n} R\right)}\left(x_{\ell}\right)\left|\gamma_{\text {red }}^{(k)}\right|\left(\mathrm{d}\left(x_{2}, \ldots, x_{k}\right)\right) \\
& =C \cdot\left|W_{n}\right|\left|\gamma_{\text {red }}^{(k)}\right|\left(\left(b\left(u_{1}, b_{n} R\right)\right)^{\ell-1} \times\left(\mathbb{R}^{d}\right)^{k-\ell}\right)
\end{aligned}
$$

for the absolute value of the term (3), where $C$ and $R$ do not depend on $n$. Now Condition $\gamma\left(\left(\boldsymbol{u}_{\boldsymbol{i} \boldsymbol{k}}\right)_{k=1}^{\ell-1}, \infty\right)$ yields the asymptotic order $\mathcal{O}\left(b_{n}^{(\ell-1) d}\left|W_{n}\right|\right)$ of this term. For an integral taken with respect to $\gamma^{(\ell)}$ the continuity of the $\ell$ th-order product density in $u_{i}$, $i=1, \ldots, q$, and Lebesgue's dominated convergence theorem again yield the asymptotic $\operatorname{order} \mathcal{O}\left(b_{n}^{(\ell-1) d}\left|W_{n}\right|\right)$.

Altogether we have

$$
\Gamma_{k}\left(a_{1} \Delta_{n}\left(\varrho^{(\ell)}, u_{1}\right)+\ldots+a_{q} \Delta_{n}\left(\varrho^{(\ell)}, u_{q}\right)\right)=\mathcal{O}\left(\left(b_{n}^{(\ell-1) d}\left|W_{n}\right|\right)^{1-k / 2}\right)
$$

as $n \rightarrow \infty$. Hence the cumulants of order three and higher converge to zero as $n \rightarrow \infty$ which implies the claimed normal convergence. The weak convergence of

$$
\sum_{i=1}^{q} \frac{\left(\Delta_{n}\left(\varrho^{(\ell)}, u_{i}\right)\right)^{2}}{\sigma_{i i}}
$$

to a $\chi^{2}$-distributed random variable with $q$ degrees of freedom follows immediately by the Continuous Mapping Theorem.

Like before, Condition $\gamma\left(\left(u_{i k}\right)_{k=1}^{\ell-1}, \infty\right)$ in the above theorem can be replaced by Condition $\gamma\left(\left(\boldsymbol{u}_{\boldsymbol{i} \boldsymbol{k}}\right)_{\boldsymbol{k}=\mathbf{1}}^{\ell-1}, \boldsymbol{p}\right)$ and $\left(b_{n}^{(\ell-1) d}\right)^{p}\left|W_{n}\right|^{p-2} \underset{n \rightarrow \infty}{\longrightarrow} \infty$ for some $p \geq 3$.

The following corollary generalizes Corollary 3.5.

Corollary 5.4. Let $\Psi \sim P$ be a $\mathrm{B}_{\infty}$-mixing $P P$ in $\mathbb{R}^{d}$ with intensity $\lambda$ and $\ell$ th-order product density $\varrho^{(\ell)}, \ell \geq 2$. Let $\left(W_{n}\right),\left(b_{n}\right)$, and $k_{d(\ell-1)}$ satisfy Condition $\mathbf{W b k}(\boldsymbol{d}(\ell-1))$ and let $b_{n}^{2+(\ell-1) d}\left|W_{n}\right| \underset{n \rightarrow \infty}{\longrightarrow} 0$. Let $u_{1}, \ldots, u_{q} \in \mathbb{R}^{d(\ell-1)}$ with $u_{i}=\left(u_{i k}\right)_{k=1}^{\ell-1} \in \mathbb{R}^{d(\ell-1)}$ be chosen such that $u_{i} \neq u_{j}$ for all $i, j \in\{1, \ldots, q\}, u_{i j} \neq o$ and $u_{i j} \neq \pm u_{i k}$ for all $i \in\{1, \ldots, q\}$ and $j, k \in\{1, \ldots, \ell-1\}$ with $j \neq k$. Let $\varrho^{(\ell)}$ be Lipschitz-continuous in $u_{i}$ for all $i=1, \ldots, q$. Let Condition $\gamma\left(\left(\boldsymbol{u}_{\boldsymbol{i k}}\right)_{\boldsymbol{k}=\mathbf{1}}^{\ell-1}, \infty\right)$ be satisfied and let the cumulant densities up to order $2 \ell$ exist and satisfy Condition $\mathbf{c}_{\ell}\left(\left(\boldsymbol{u}_{\boldsymbol{i} \boldsymbol{k}}, \boldsymbol{u}_{\boldsymbol{j} \boldsymbol{k}}\right)_{\boldsymbol{k}=1}^{\ell-1}\right)$ for all $i, j=1, \ldots, q$.

Then we have

$$
\left(\frac{2 \sqrt{b_{n}^{(\ell-1) d}\left|W_{n}\right|}}{\left\|k_{d(\ell-1)}\right\|}\left(\sqrt{\varrho_{n}^{(\ell)}\left(u_{i}\right)}-\sqrt{\lambda \varrho^{(\ell)}\left(u_{i}\right)}\right)\right)_{i=1}^{q} \underset{n \rightarrow \infty}{\stackrel{\mathrm{d}}{\longrightarrow}} \mathcal{N}\left(0_{q}, I_{q}\right) .
$$

Furthermore we have

$$
\frac{4 b_{n}^{(\ell-1) d}\left|W_{n}\right|}{\left\|k_{d(\ell-1)}\right\|^{2}} \sum_{i=1}^{q}\left(\sqrt{\hat{\varrho}_{n}^{(\ell)}\left(u_{i}\right)}-\sqrt{\lambda \varrho^{(\ell)}\left(u_{i}\right)}\right)^{2} \underset{n \rightarrow \infty}{\stackrel{\mathrm{d}}{\longrightarrow}} \chi_{q}^{2} .
$$




\section{Appendix: Indecomposable integrals}

The notion of indecomposable integrals has already been used in David and Heinrich [5]. The lemma given in this appendix can also be found in David and Heinrich [5, Lemma 5.1]. The lemma states that the $k$ th cumulant of certain random variables is a sum of integrals that are indecomposable, in the sense that they cannot be represented as a product of two integrals. The rigorous definition of decomposability is as follows.

Let $f_{i}:\left(\mathbb{R}^{d}\right)^{p_{i}} \rightarrow \mathbb{R}$ be fixed measurable functions, let $k \in \mathbb{N}$ and $p_{i} \in \mathbb{N}$ with $i \in I=$ $\{1, \ldots, k\}$ be fixed and set

$$
\Psi^{\left(p_{i}\right)}\left(f_{i}\right):=\sum_{x_{1}, \ldots, x_{p_{i}} \in \Psi} f_{i}\left(x_{1}, \ldots, x_{p_{i}}\right)
$$

Let $\mathbb{E}\left[\left|\Psi^{\left(p_{i}\right)}\left(f_{i}\right)\right|^{k}\right]<\infty$ for all $i \in I$. We will now find a representation of the mixed moment $M\left(\Psi^{\left(p_{1}\right)}\left(f_{1}\right), \ldots, \Psi^{\left(p_{k}\right)}\left(f_{k}\right)\right):=\mathbb{E}\left[\prod_{i=1}^{k} \Psi^{\left(p_{i}\right)}\left(f_{i}\right)\right]$ as a sum of integrals defined as follows.

For arbitrary $T \subseteq I, q \in\left\{1, \ldots, p_{T}\right\}$ with $p_{T}:=\sum_{i \in T} p_{i}, r \in\{1, \ldots, q\}$, and decompositions $\mathcal{P}_{T}=\left\{P_{1}, \ldots, P_{q}\right\}$ of $\left\{1, \ldots, p_{T}\right\}$ and $\mathcal{Q}=\left\{Q_{1}, \ldots, Q_{r}\right\}$ of $\{1, \ldots, q\}$ we define the integral

$$
\begin{aligned}
& I_{\mathcal{P}_{T}, \mathcal{Q}}\left(f_{i}: \quad i \in T\right) \\
& :=\int_{\left(\mathbb{R}^{d}\right)^{q}} \prod_{b=1}^{q} \prod_{a \in P_{b}} \mathbb{1}_{\left\{x_{a}=z_{b}\right\}} f_{i_{1}}\left(x_{1}, \ldots, x_{p_{i_{1}}}\right) \\
& \quad \quad \times f_{i_{2}}\left(x_{p_{i_{1}}+1}, \ldots, x_{p_{i_{1}}+p_{i_{2}}}\right) \cdot \ldots \cdot f_{i_{\# T}}\left(x_{\sum_{j=1}^{\# T-1} p_{i_{j}}+1}, \ldots, x_{p_{T}}\right) \prod_{c=1}^{r} \gamma^{\left(\# Q_{c}\right)}\left(\mathrm{d} \mathbf{z}_{Q_{c}}\right),
\end{aligned}
$$

where $\left\{i_{1}, \ldots, i_{\# T}\right\}=T$ with $1 \leq i_{1}<i_{2}<\ldots<i_{\# T} \leq k$ and $\mathbf{z}_{Q_{c}}=\left(z_{q}\right)_{q \in Q_{c}}$. The elements of a set $P_{b}$ are the indices of the arguments of the functions $f_{i_{1}}, \ldots, f_{i_{\# T}}$ that are identical and distinct from all the arguments in every other set $P_{c} \neq P_{b}$. In the above-mentioned integral this is indicated by the term $\prod_{b=1}^{q} \prod_{a \in P_{b}} \mathbb{1}_{\left\{x_{a}=z_{b}\right\}}$. For the special case $T=I$ we have

$$
\begin{aligned}
& I_{\mathcal{P}_{I}, \mathcal{Q}}\left(f_{1}, \ldots, f_{k}\right) \\
& \quad=\int_{\left(\mathbb{R}^{d}\right)^{q}} \prod_{b=1}^{q} \prod_{a \in P_{b}} \mathbb{1}_{\left\{x_{a}=z_{b}\right\}} f_{1}\left(x_{1}, \ldots, x_{p_{1}}\right) \cdot \ldots \cdot f_{k}\left(x_{\sum_{i=1}^{k-1} p_{i}+1}, \ldots, x_{p_{I}}\right) \prod_{c=1}^{r} \gamma^{\left(\# Q_{c}\right)}\left(\mathrm{d} \mathbf{z}_{Q_{c}}\right) .
\end{aligned}
$$

Now the mixed moment $M\left(\Psi^{\left(p_{1}\right)}\left(f_{1}\right), \ldots, \Psi^{\left(p_{k}\right)}\left(f_{k}\right)\right)$ can be represented as

$$
\begin{aligned}
& M\left(\Psi^{\left(p_{1}\right)}\left(f_{1}\right), \ldots, \Psi^{\left(p_{k}\right)}\left(f_{k}\right)\right) \\
& =\sum_{\substack{q=1 \\
p_{I}}} \sum_{\substack{P_{1} \cup \ldots \cup P_{q} \\
=\left\{1, \ldots, p_{I}\right\}}} \int_{\left(\mathbb{R}^{d}\right)^{q}} \prod_{b=1}^{q} \prod_{\substack{a \in P_{b}\\
}} \mathbb{1}_{\left\{x_{a}=z_{b}\right\}} \\
& \quad \times f_{1}\left(x_{1}, \ldots, x_{p_{1}}\right) \cdot \ldots \cdot f_{k}\left(x_{\sum_{i=1}^{k-1} p_{i}}, \ldots, x_{p_{I}}\right) \alpha^{(q)}\left(\mathrm{d}\left(z_{1}, \ldots, z_{q}\right)\right)
\end{aligned}
$$




$$
\begin{aligned}
&=\sum_{q=1}^{p_{I}} \sum_{\substack{P_{1} \cup \ldots \cup P_{q} \\
=\{1, \ldots, p\}}} \sum_{r=1}^{q} \sum_{\substack{Q_{1} \cup \ldots \cup Q_{r} \\
=\{1, \ldots, q\}}} \int_{\left(\mathbb{R}^{d}\right)^{q}} \prod_{b=1}^{q} \prod_{a \in P_{b}} \mathbb{1}_{\left\{x_{a}=z_{b}\right\}} \\
& \times f_{1}\left(x_{1}, \ldots, x_{p_{1}}\right) \cdot \ldots \cdot f_{k}\left(x_{\sum_{i=1}^{k-1} p_{i}+1}, \ldots, x_{p_{I}}\right) \prod_{c=1}^{r} \gamma^{\left(\# Q_{c}\right)}\left(\mathrm{d} \mathbf{z}_{Q_{c}}\right),
\end{aligned}
$$

see Krickeberg [14]. With the above notation we have

$$
M\left(\Psi^{\left(p_{1}\right)}\left(f_{1}\right), \ldots, \Psi^{\left(p_{k}\right)}\left(f_{k}\right)\right)=\sum_{q=1}^{p_{I}} \sum_{\substack{P_{1} \cup \ldots \cup P_{q} \\=\left\{1, \ldots, p_{I}\right\}}} \sum_{r=1}^{q} \sum_{\substack{Q_{1} \cup \ldots \cup Q_{r} \\=\{1, \ldots, q\}}} I_{\mathcal{P}_{I}, \mathcal{Q}}\left(f_{1}, \ldots, f_{k}\right) .
$$

Let $T=\left\{T_{1}, T_{2}\right\}$ be a decomposition of $I=\{1, \ldots, k\}$. An integral $I_{\mathcal{P}_{I}, \mathcal{Q}}\left(f_{1}, \ldots, f_{k}\right)$ is decomposable with respect to the decomposition $T=\left\{T_{1}, T_{2}\right\}$ if there exist a decomposition $\mathcal{P}^{(1)}$ of $\left\{1, \ldots, p_{T_{1}}\right\}$, a decomposition $\mathcal{P}^{(2)}$ of $\left\{1, \ldots, p_{T_{2}}\right\}, q_{1} \in\left\{1, \ldots, p_{T_{1}}\right\}$ and $q_{2} \in\left\{1, \ldots, p_{T_{2}}\right\}$ with $q_{1}+q_{2}=q$, and decompositions $\mathcal{Q}^{(1)}$ of $\left\{1, \ldots, q_{1}\right\}$ and $\mathcal{Q}^{(2)}$ of $\left\{1, \ldots, q_{2}\right\}$ such that

$$
I_{\mathcal{P}_{I}, \mathcal{Q}}\left(f_{1}, \ldots, f_{k}\right)=I_{\mathcal{P}_{T_{1}}, \mathcal{Q}^{(1)}}\left(f_{i}: i \in T_{1}\right) \cdot I_{\mathcal{P}_{T_{1}}, \mathcal{Q}^{(2)}}\left(f_{i}: i \in T_{2}\right) .
$$

An integral is called decomposable if there exists a nontrivial decomposition of $I$ such that this integral is decomposable with respect to this decomposition. An integral which is not decomposable with respect to any nontrivial decomposition is called indecomposable.

The following lemma is the key tool for the proof of Theorem 3.3. It gives a representation of the $k$ th cumulant of certain random variables as a sum of indecomposable integrals. Let $\Gamma_{k}(X)$ denote the $k$ th cumulant of a real-valued random variable $X$ and $\operatorname{Cum}_{k}\left(X_{1}, \ldots, X_{k}\right)$ denote the mixed cumulant of a random vector $X=\left(X_{1}, \ldots, X_{k}\right)^{\prime} \in \mathbb{R}^{k}, k \geq 1$.

Lemma. Let $\Psi \sim P$ be a $P P$ in $\mathbb{R}^{d}$. Let $j, k \in \mathbb{N}$ be fixed, let $C_{i} \in \mathbb{R}$ be constants for $i=1, \ldots, j$, and set

$$
\Psi^{\left(p_{i}\right)}\left(f_{i}\right)=\sum_{x_{1}, \ldots, x_{p_{i}} \in \Psi} f_{i}\left(x_{1}, \ldots, x_{p_{i}}\right)
$$

where $f_{i}:\left(\mathbb{R}^{d}\right)^{p_{i}} \rightarrow \mathbb{R}$ is a fixed measurable function with $p_{i} \in \mathbb{N}$, for $i=1, \ldots, j$. Let $\mathbb{E}\left[\left|\Psi^{\left(p_{i}\right)}\left(f_{i}\right)\right|^{k}\right]<\infty$ for all $i=1, \ldots, j$. Then we have

$$
\Gamma_{k}\left(\sum_{i=1}^{j} C_{i} \Psi^{\left(p_{i}\right)}\left(f_{i}\right)\right)=\sum_{\substack{k_{1}+\ldots+k_{j}=k \\ k_{1}, \ldots, k_{j} \geq 0}} \frac{k !}{k_{1} ! \cdot \ldots \cdot k_{j} !} C_{1}^{k_{1}} \cdot \ldots \cdot C_{j}^{k_{j}} \mu_{k_{1}, \ldots, k_{j}}^{*},
$$

where

$$
\mu_{k_{1}, \ldots, k_{j}}^{*}:=\left(\sum_{q=1}^{p_{k_{1}, \ldots, k_{j}}} \sum_{\substack{P_{1} \cup \ldots \cup P_{q} \\=\left\{1, \ldots, p_{\left.k_{1}, \ldots, k_{j}\right\}}\right.}}^{q} \sum_{r=1}^{q} \sum_{\substack{Q_{1} \cup \ldots \cup Q_{r} \\=\{1, \ldots, q\}}}\right)^{*} I_{\mathcal{P}_{I}, \mathcal{Q}}(\underbrace{f_{1}, \ldots, f_{1}}_{k_{1}}, \ldots, \underbrace{f_{j}, \ldots, f_{j}}_{k_{j}})
$$

and $p_{k_{1}, \ldots, k_{j}}=\sum_{i=1}^{j} p_{i} k_{i}$. The summation $(\cdot)^{*}$ is taken only over the indecomposable integrals.

The proof of the above lemma is given in David and Heinrich [5]. 


\section{Acknowledgements}

This work was supported by the Deutsche Forschungsgemeinschaft through research grants HE 3055/3-1 and HE 3055/3-2.

\section{References}

[1] Baddeley, A., Turner, R., Møller, J., and Hazelton, M. (2005). Residual analysis for spatial point processes (with discussion). Journal of the Royal Statistical Society, Series $B, 67(5): 617-666$.

[2] Brillinger, D.R. (1975). Statistical inference for stationary point processes. In Puri, M. L.(ed), Stochastic Processes and Related Topics, Volume 1, Proceedings of the Summer Research Institute on Statistical Inference for Stochastic Processes, pp. 55-99. Academic Press, New York.

[3] Cressie, N.A.C. (1993). Statistics for Spatial Data. Wiley \& Sons, New York.

[4] Daley, D.J. and Vere-Jones, D. (2008). An Introduction to the Theory of Point Processes. Vol. II: Genereral Theory and Structure. Springer, New York.

[5] David, S. and Heinrich, L. (2010). Central limit theorem for the integrated squared error of the empirical second-order product density and goodness-of-fit tests for stationary point processes. submitted, see also Preprint No. 17-2010, Institut für Mathematik, Universität Augsburg. http://opus.bibliothek.uni-augsburg.de/volltexte/2010/1674/.

[6] Diggle, P.J. (2003). Statistical Analysis of Spatial Point Patterns. 2nd ed. Arnold, London.

[7] Heinrich, L. (1988). Asymptotic Gaussianity of some estimators for reduced factorial moment measures and product densities of stationary Poisson cluster processes. Statistics, 19:87-106.

[8] Heinrich, L. and Liebscher, E. (1997). Strong convergence of kernel estimators for product densities of absolutely regular point processes. Nonparametric Statistics, 8:65-96.

[9] Heinrich, L. and Schmidt, V. (1985). Normal convergence of multidimensional shot noise and rates of this convergence. Advances in Applied Probability, 17:709-730.

[10] Illian, J., Penttinen, A., Stoyan, H., and Stoyan, D. (2008). Statistical Analysis and Modelling of Spatial Point Patterns. Wiley \& Sons, Chichester.

[11] Jolivet, E. (1981). Central limit theorem and convergence of empirical processes for stationary point processes. In Point processes and Queueing Problems. P. Bartfai and J. Tomko (eds), North-Holland, Amsterdam, 117 - 161. 
[12] Jolivet, E. (1984). Upper bounds of the speed of convergence of moment density estimators for stationary point processes. Metrika, 31:349-360.

[13] Karr, A.F. (1986). Point processes and their statistical inference. Marcel Dekker, New York.

[14] Krickeberg, K. (1982). Processus ponctuels en statistique. In École d'Éte de Probabilités de Saint-Flour X - 1980, volume 929 of Lecture Notes in Mathematics, pp. 205-313. Springer, Berlin.

[15] Leonov, V.P. and Shiryaev, A.N. (1959). On a method of calculation of semi-invariants. Theory of Probability and its Applications, 4(3):319-329.

[16] Stoyan, D., Kendall, W.S., and Mecke, J. (1995). Stochastic Geometry and Its Applications. 2nd ed. Wiley \& Sons, New York.

[17] Stoyan, D. and Stoyan, H. (2000). Improving ratio estimators of second order point process characteristics. Scandinavian Journal of Statistics, 27:641-656. 